\title{
The Promotion of Human Neural Stem Cells Adhesion Using Bioinspired Poly(norepinephrine) Nanoscale Coating
}

\author{
Minah Park, ${ }^{1}$ Mikyung Shin, ${ }^{2}$ Eunmi Kim, ${ }^{1}$ Slgirim Lee, ${ }^{1}$ Kook In Park, ${ }^{3}$ \\ Haeshin Lee, ${ }^{2,4}$ and Jae-Hyung Jang ${ }^{1}$ \\ ${ }^{1}$ Department of Chemical and Biomolecular Engineering, Yonsei University, 262 Seongsanno, Seodaemun-gu, \\ Seoul 120-749, Republic of Korea \\ ${ }^{2}$ The Graduate School of Nanoscience and Technology, KAIST, Daejeon 305-701, Republic of Korea \\ ${ }^{3}$ Department of Pediatrics, College of Medicine, Yonsei University, Seoul 120-749, Republic of Korea \\ ${ }^{4}$ Department of Chemistry, The Graduate School of Nanoscience and Technology, \\ Korea Advanced Institute of Science and Technology (KAIST), 291 University Road, Daejeon 305-701, Republic of Korea \\ Correspondence should be addressed to Haeshin Lee; haeshin@kaist.ac.kr and Jae-Hyung Jang; j-jang@yonsei.ac.kr
}

Received 21 November 2013; Accepted 12 February 2014; Published 7 April 2014

Academic Editor: Jingxia Wang

Copyright (C) 2014 Minah Park et al. This is an open access article distributed under the Creative Commons Attribution License, which permits unrestricted use, distribution, and reproduction in any medium, provided the original work is properly cited.

\begin{abstract}
The establishment of versatile biomaterial interfaces that can facilitate cellular adhesion is crucial for elucidating the cellular processes that occur on biomaterial surfaces. Furthermore, biomaterial interfaces can provide physical or chemical cues that are capable of stimulating cellular behaviors by regulating intracellular signaling cascades. Herein, a method of creating a biomimetic functional biointerface was introduced to enhance human neural stem cell (hNSC) adhesion. The hNSC-compatible biointerface was prepared by the oxidative polymerization of the neurotransmitter norepinephrine, which generates a nanoscale organic thin layer, termed poly(norepinephrine) (pNE). Due to its adhesive property, pNE resulted in an adherent layer on various substrates, and pNE-coated biointerfaces provided a highly favorable microenvironment for hNSCs, with no observed cytotoxicity. Only a 2-hour incubation of hNSCs was required to firmly attach the stem cells, regardless of the type of substrate. Importantly, the adhesive properties of pNE interfaces led to micropatterns of cellular attachment, thereby demonstrating the ability of the interface to organize the stem cells. This highly facile surface-modification method using a biomimetic pNE thin layer can be applied to a number of suitable materials that were previously not compatible with hNSC technology.
\end{abstract}

\section{Introduction}

Creating biomaterial interfaces that are capable of inducing intimate interactions with cells is a crucial step to elucidating the sophisticated cellular processes occurring on biomaterial surfaces in numerous biomedical applications. The surface properties of cell-material interfaces can influence cellular events related to cellular attachment, patterning, proliferation, and differentiation $[1,2]$. Indeed, interfaces contacting the cellular membrane not only influence cellular morphology but also function as intermediates that can supplement the molecular signals that direct various biological processes $[3,4]$. However, the generation of appropriate interfacial environments that can effectively regulate intracellular signaling cascades and control cellular fates remains a challenge. One solution is the use of a material that is capable of functioning as an interface, with the crucial requirement that the material be able to promote cellular attachment on its surface.

Numerous surface-modification methods have been employed to enhance cellular adhesion. The majority of modification approaches have attempted to interact with target cells through the chemical tethering or physical adsorption of particular biological moieties, such as integrin ligands [5], specialized peptides [6], growth factors [7], or functionalized chemicals [8], onto the modified surfaces. Additionally, modifications of surface properties, as achieved by producing self-assembled monolayers or by engaging organosilane chemistry, have been employed to regulate interactions with 
cells [9]. The manipulation of surface properties, resulting in variations in cellular attachment and cellular patterning, has been attempted using various lithographic techniques, including writing-based lithography [10-12] or surface graft polymerization approaches [13]. Additionally, the topographical changes of three-dimensional cell-culture structures [14], well-defined structural patterns [15], and surface rigidity [16] directly influenced cell adhesion on substrates and survivals as well. However, several issues, such as the requirement of organic solvents, difficulty in the large-scale production of modified surfaces, requirement of expensive instruments, or time-consuming labor requirements, may limit the extensive utilization of these techniques in various applications.

The adhesive properties of catecholamine compounds, as characterized by the sticky characteristics of the foot proteins of marine mussels, have been reported to be useful sources for universally modifying numerous surfaces [ 9 , 17, 18]. The adhesive properties of catecholamines, representatively as poly(dopamine), have helped to realize the efficient immobilization of biomolecules onto surfaces with various chemistries [18]. A surface can also be functionalized by dissolving biomolecules with catecholamine, particularly poly(dopamine), during nanoscale organic thin layer formation, ultimately leading to the surface immobilization of biomolecules. Furthermore, secondary modifications can be performed through surface-initiated polymerization and biomineralization [19]. Importantly, the facile approach of using a catecholamine group as a coating agent not only allows flexibility in the selection of the substrate materials but is also an inexpensive and ecofriendly process [20]. Lastly, the spatial arrangement of the surface adhesiveness may result in the patterned regulation of cellular behaviors, including differentiation, proliferation, and migration [17, 21].

Recently, the neurotransmitter norepinephrine has been shown to functionalize virtually any material surface when it is oxidatively polymerized to produce poly(norepinephrine) (pNE) $[22,23]$. The pNE coating layer exhibits unique properties, such as ring-opening polymerization and the storage/release of nitric oxide [24]. Nonetheless, the capability of facilitating stem cell adhesion onto a pNE layer has not been investigated to date. In this study, we demonstrate that a pNE layer can be formed on various substrates (poly(dimethylsiloxane) (PDMS), poly(tetrafluoroethylene) (PTFE), tissue culture polystyrene (TCP), and glass), resulting in the dramatic enhancement of the adhesion of hNSCs isolated from human fetal brain. Additionally, the hNSC viability was not compromised, and cellular and cytoskeletal morphology were assessed. Considering the very low adhesive properties of hNSCs, our study shows that the formation of a pNE nanolayer can be a good platform for the stable culture of hNSCs, which may support the ex vivo expansion of therapeutic stem cells.

\section{Experimental Section}

2.1. Cell Culture. Human fetal neural stem cells (hNSC, 13 weeks of gestational age) were utilized to analyze cellular adhesion, patterning, and proliferation on pNE interfaces formed on various substrates and transduction efficiencies. hNSCs were derived from the telencephalon (HFT13) as previously described [25] and cultured in Dulbecco's modified Eagle's medium/F12 (Invitrogen, Carlsbad, CA, USA) containing N-2 supplement, $8 \mathrm{mg} / \mathrm{mL}$ heparin (Sigma-Aldrich, $\mathrm{St}$ Louis, MO, USA), $20 \mathrm{ng} / \mathrm{mL}$ fibroblast growth factor-2 (FGF2; R\&D Systems, Minneapolis, MN, USA), and $10 \mathrm{ng} / \mathrm{mL}$ leukemia inhibitory factor (LIF; Chemicon, Temecula, CA, USA).

2.2. Substrate Preparation and $p N E$ Coating. Four different substrates, including glass (Marienfeld $\mathrm{GmbH}$, Germany), poly(dimethylsiloxane) (PDMS; Sylgard 184, Dow Corning, MI, U.S.A), poly(tetrafluoroethylene) (PTFE; Hanmi Rubber \& Plastics, Korea), and tissue culture polystyrene surfaces, were sterilized in $70 \%$ ethanol and rinsed with sterile-distilled water prior to $\mathrm{pNE}$ modification. PDMS was prepared using a SYLGARD 184 silicone elastomer kit (Dow Corning Corporation, Midland, MI, USA) according to the manufacturer's guidelines. Each substrate was immersed in $1 \mathrm{~mL}$ of $10 \mathrm{mM}$ Tris ( $\mathrm{pH}$ 8.5) solution containing $2 \mathrm{mg} \mathrm{L-(-)-norepinephrine}$ in $1 \mathrm{~mL}$ of $10 \mathrm{mM}$ Tris (pH 8.5) at room temperature for 24 hours. During this procedure, norepinephrine monomers polymerize to form pNE interfaces on the surface of each substrate. The coated substrates were additionally sterilized with $70 \%$ ethanol, rinsed twice with sterile-distilled water, and air-dried. The surface wettability and morphology of each pNE-coated surface were determined by contact angle measurements (CAM 101, KSV Instruments Ltd., Espoo, Finland) and atomic force measurements (AFM) (XE-BIO AFM, Parks Systems, Korea), respectively. Additionally, the root mean square (RMS) roughness (Rq) of the modified surfaces was measured by the AFM analysis.

2.3. Cellular Attachment on pNE-Coated Substrate. Each substrate coated with the pNE interface was placed in the wells of a 48-well tissue culture plate using silicone grease, and hNSCs were seeded at a cell density of $10^{4}$ cells $/ 10 \mu \mathrm{L}$. The cells were incubated for an hour at $37^{\circ} \mathrm{C}$ and $5 \% \mathrm{CO}_{2}$, and $200 \mu \mathrm{L}$ of medium was subsequently added to feed the cells on each substrate. An hour later, the substrates with cells were placed in new wells, and the cells were trypsinized from the surface to quantify the number of cells that had successfully attached to the surface during the short culture period ( $\sim 1$ hour). The number of cells was counted using a hemocytometer.

2.4. Morphologies of Cells Attached on pNE-Coated Substrate. The cytoskeletal morphology of the cells adhered to each pNE-coated substrate was assessed by visualizing filamentous actin, which was stained using rhodamine-labeled phalloidin (1:50 dilution in PBS) (Invitrogen, Carlsbad, CA, USA); the nuclei were counterstained using DAPI (Vector Laboratories, Burlingame, CA). Briefly, the coating of each substrate with the pNE interface and cellular attachment was performed according to the aforementioned methodology, and the remaining cells on each substrate were cultured for 2 days prior to the histological analysis. The cells were subsequently 
<smiles>NCC(O)c1ccc(O)c(O)c1</smiles>

Norepinephrine $\left(\mathrm{C}_{8} \mathrm{H}_{11} \mathrm{NO}_{3}\right)$

(a)

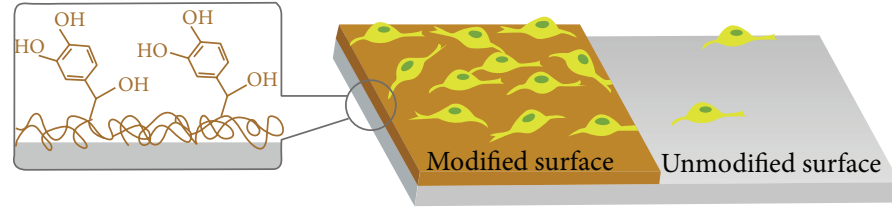

Increased cell adhesion on the modified surface

(b)

FIGURE 1: Surface modification using pNE. (a) Chemical structure of norepinephrine. (b) Schematic illustration of the pNE-coated biointerfaces onto which hNSCs are adhered.

fixed in 4\% paraformaldehyde (PFA) for 20 minutes and blocked with $5 \%$ goat serum supplemented with $0.1 \%$ Triton $\mathrm{X}-100$. The cells were incubated with rhodamine-labeled phalloidin (Invitrogen, Carlsbad, CA, USA) for 30 minutes in the dark, rinsed three times with PBS, and counterstained with DAPI for 30 minutes. The fluorescence images of both the cytoskeletal morphology and nucleus of the cells were acquired using confocal microscopy (LSM 700, Carl Zeiss).

2.5. Cellular Cytotoxicity Assay on pNE-Coated Substrates. To determine the cytotoxicity of the pNE interfaces, the metabolic activities of hNSCs cultured on each pNE-coated surface were analyzed using a WST-1 assay kit (Roche Applied Science, Indianapolis, IN) according to the manufacturer's protocol. After 2, 4, and 6 days of culture on each pNE-coated substrate, a 0.1 volume of WST-1 solution (as a proportion of the culture medium) was directly added to each well and incubated for an additional 2 hours at $37^{\circ} \mathrm{C}$. The supernatants were collected, and the colorimetric changes at $440 \mathrm{~nm}$ were measured using a spectrophotometer (NanoDrop 2000, Thermo Scientific, West Palm Beach, FL, USA).

2.6. Macro- or Microscale Cellular Patterning on pNE-Coated Substrates. To produce cellular patterns with macroscales $(\sim \mathrm{mm})$, specific patterns of the pNE interfaces, such as a "Y" shape, were generated by simply drawing the shape with the pNE solution using a micropipette and incubating at $37^{\circ} \mathrm{C}$ overnight to form the patterned interface. Tissue culture plates were employed as a representative of the cellular patterning due to the ease of analyzing the cellular patterns with a microscope. After washing with PBS, the cells were subsequently seeded at $6 \times 10^{6}$ cells $/ \mathrm{mL}$; after 2 hours, the cells barely attached to the substrate were removed by gently washing with PBS. After being fixed with 4\% PFA, the remaining cells were stained with Hoechst dye (Sigma-Aldrich, St Louis, MO, USA). The images of the cellular patterns were acquired using a fluorescence microscope (Nikon TE2000E2, Nikon, Japan). PDMS stamping technology was utilized to generate cellular patterns on a microscale $(\sim 100 \mu \mathrm{m})$. Briefly, a PDMS micropattern with line alignments was initially generated with a specified dimension (ridge, $100 \mu \mathrm{m}$; groove, $200 \mu \mathrm{m}$; and depth, $120 \mu \mathrm{m}$ ). The PDMS stamp was immersed into a pNE solution $(2 \mathrm{mg} / \mathrm{mL}$ of norepinephrine in $10 \mathrm{mM}$ Tris at $\mathrm{pH} 8.5$ ), which was prepolymerized for 24 hours, placed on the tissue culture plate, and pressed manually for 2 minutes. The unbound $\mathrm{pNE}$ interfaces on the tissue culture plates were removed by rinsing twice with PBS, and cells at $6 \times 10^{6}$ cells $/ \mathrm{mL}$ were seeded for attachment to the $\mathrm{pNE}$ interfaces on the glass. As with the macroscale patterning, the cells barely attached to the pNE interface were removed by washing with PBS after 2 hours, and the nuclei of the remaining cells were stained with DAPI and imaged using a fluorescence microscope (Nikon TE2000E2, Nikon, Japan) to visualize the cellular alignment and the pNE patterns generated by the PDMS stamp.

2.7. Statistics. All of the experimental data are illustrated as the mean \pm the standard deviation (SD), and statistical significances were analyzed by a one-way analysis of variance (ANOVA) with a post hoc Dunnett's test using the SPSS 18.0 software package (IBM Corporation, Somers, NY, USA).

\section{Results and Discussion}

Figure 1 schematically shows the generation of biointerfaces by $\mathrm{pNE}$ coating. The chemical structure shown in the figure is catechol, which is responsible for robust adhesion onto substrates and the rapid immobilization of biological components for cell adhesion. As illustrated, the pNE layer is rich in hydroxyl groups: two hydroxyl groups from catechol and one from alkyl chain. It is known that a new intermediate component, 3,4-dihydroxybenzaldehyde (DHBA), is formed, which subsequently reacts with norepinephrine (NE) to yield DHBA-NE during the oxidative polymerization of norepinephrine. It has recently been shown that DHBA functions as a key factor contributing a conformal, smooth coating on a substrate in comparison to the well-known surface functionalization of poly(dopamine) [24]. Similar to the case of poly(dopamine), we hypothesized that $\mathrm{pNE}$ thin layers can effectively promote neural stem cell adhesion. Unlike typical mammalian cells, hNSCs do not adhere stably to tissue culture polystyrene dishes. Thus, the development of a culture platform promoting hNSC adhesion is a critical issue in the ex vivo expansion of stem cells for cell therapy. To test the functionality of $\mathrm{pNE}$ as a coating agent, a wide range of materials, including poly(tetrafluoroethylene) (PTFE), poly(dimethylsiloxane) (PDMS), glass, and tissue culture polystyrene (TCP) surfaces, were modified using the dip-coating method in an alkaline norepinephrine solution [22], as described in Figure 1(b). These substrates were chosen 


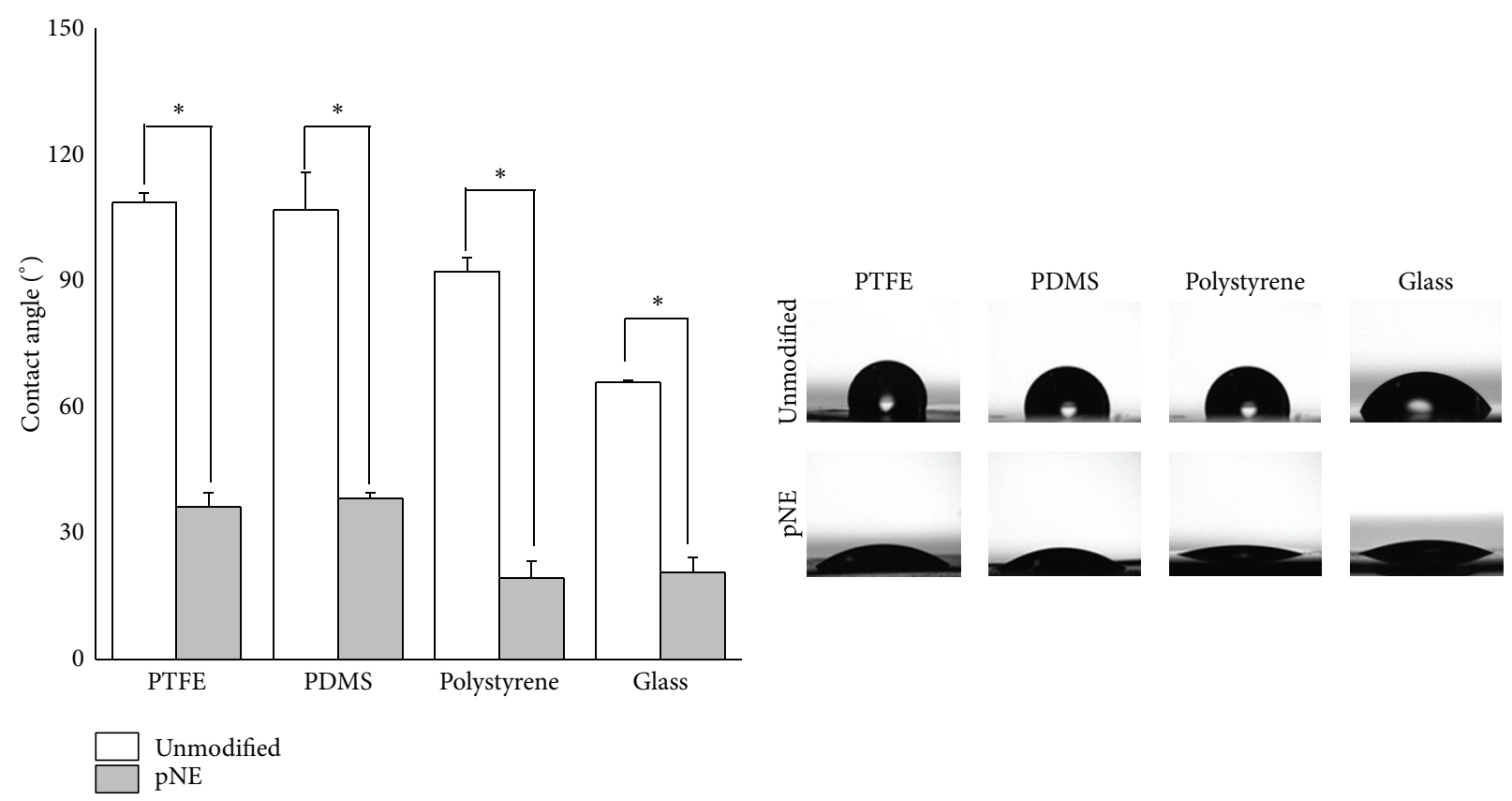

(a)
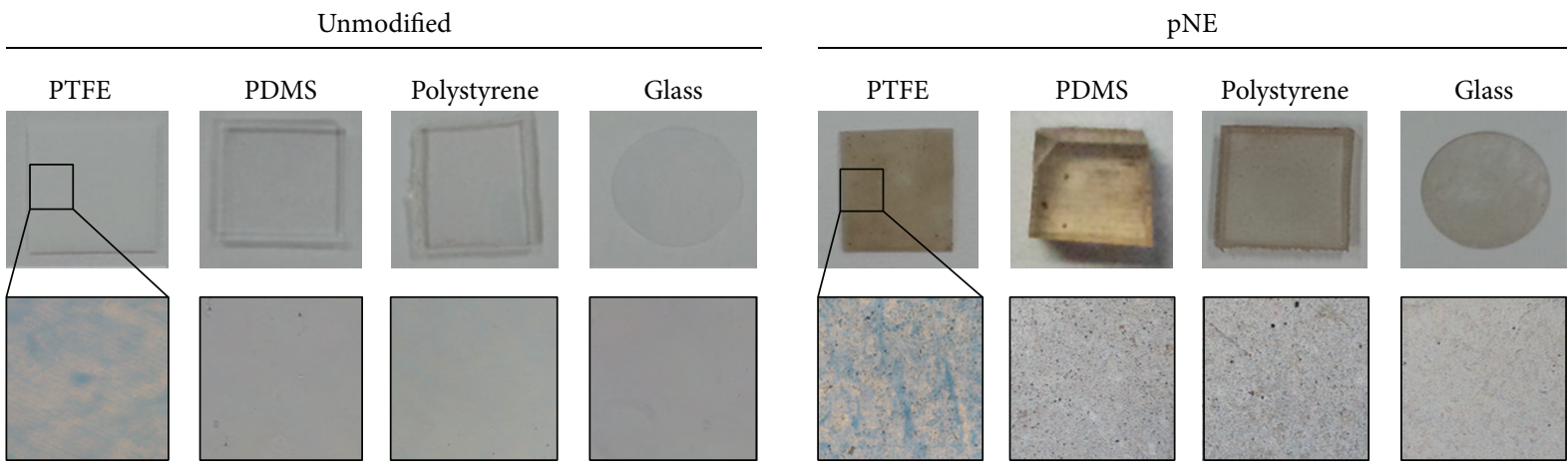

(b)
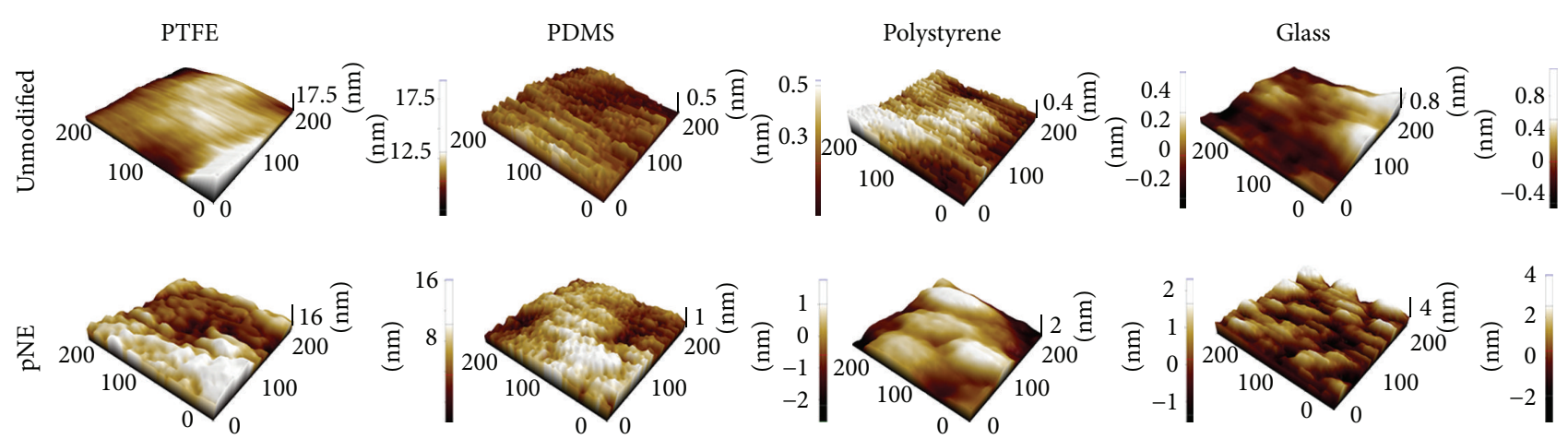

(c)

FIGURE 2: Characterization of pNE surfaces. (a) Surface wettability is measured by a goniometer. The symbol $*$ indicates a significant difference compared to each unmodified substrate $(P<0.005)$. (b) Representative digital images of pNE-coated PTFE, PDMS, glass, and TCP substrates. The dark brown colors indicate pNE coating. (c) Topographical images of pNE interfaces using AFM analysis. 


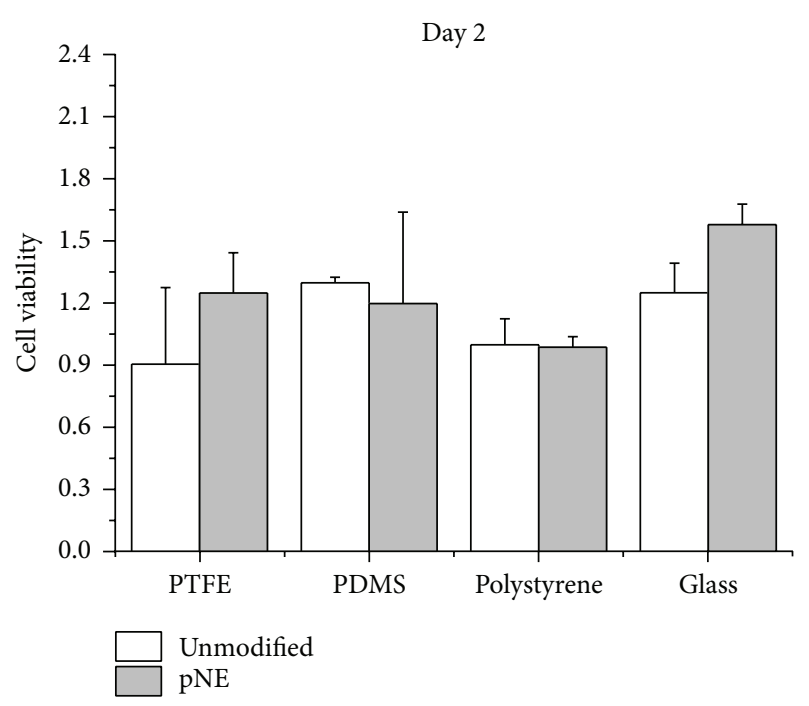

(a)

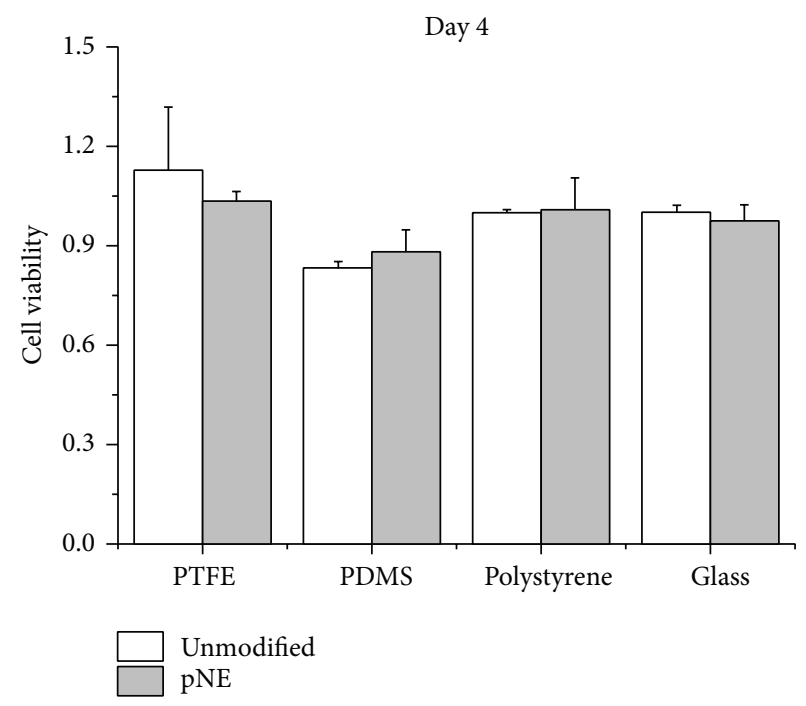

(b)

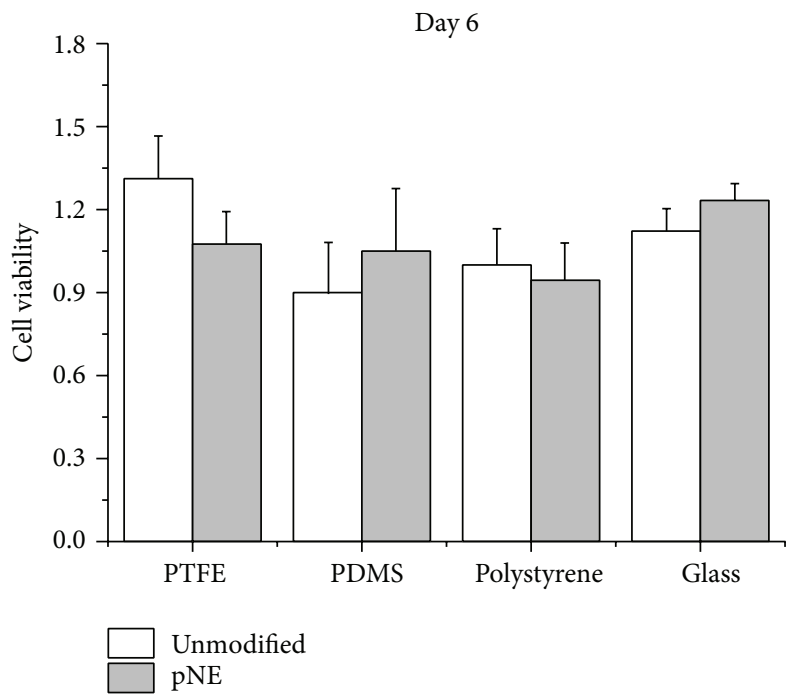

(c)

FIgURE 3: The cytotoxicity of pNE-functionalized biointerfaces. The metabolic activity of hNSCs grown on pNE interfaces at 2, 4, and 6 days was measured using WST-1 and compared to the unmodified substrates.

to demonstrate the versatility of norepinephrine, which can form interfaces on various types of materials to facilitate cellular adhesion.

The characterization of the pNE-coated surfaces revealed that the polymerization of norepinephrine (NE) in a weak alkaline solution $(\mathrm{pH} \mathrm{8.5)}$ on various materials resulted in similar properties of the exterior surfaces, regardless of the substrate type. As demonstrated in Figure 2, coating with the pNE interface allowed various substrates to acquire a similar wettability and surface morphology. Variations in the wettability of each substrate coated with pNE were detected by measuring the water-contact angles on each substrate (Figure 2(a)). Regardless of the substrate properties, the pNE coating dramatically reduced the contact angles of all the substrates, with final values ranging from $22.76^{\circ}$ to $39.86^{\circ}$.
The results were originated from the hydrophilic properties of the pNE coating, which had many hydrophilic moieties such as hydroxyl groups and amine groups. Additionally, both digital images and atomic force microscopy (AFM) analyses revealed that the substrates coated with pNE had smooth surface topographies, regardless of the substrate type (Figures 2(b) and 2(c)). Furthermore, the AFM analysis confirmed no large aggregates when each surface was modified with pNE (Figure 2(c)). Table 1 shows the root-mean-square (RMS) roughness values of the pNE interfaces, confirming significant differences from those of the unmodified substrates $(P<0.001)$. All of these pNE surface characterization results indicate its remarkable capability to induce material-independent surface modification with uniform properties. 


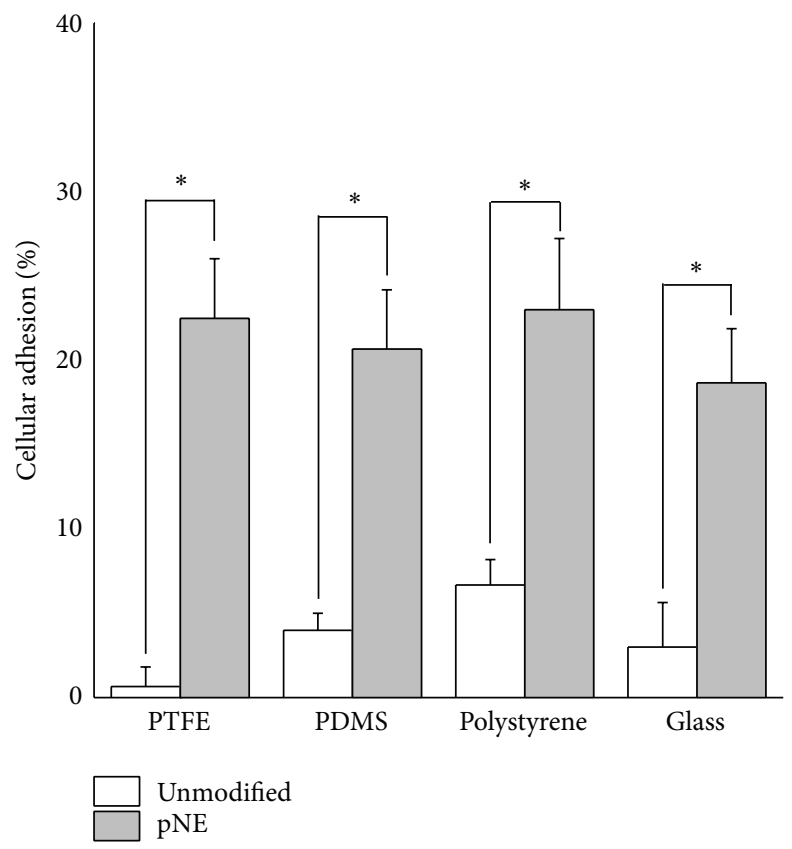

(a)
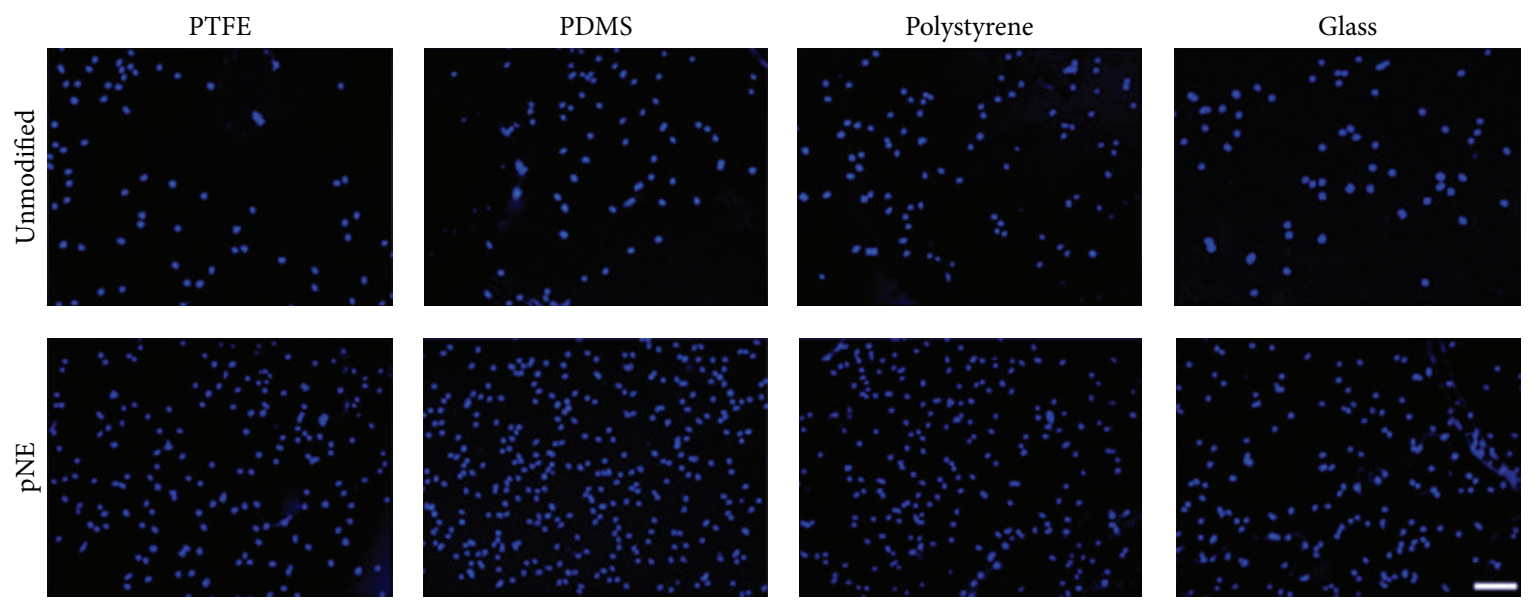

(b)

FIGURE 4: Cellular adhesion on pNE interfaces. (a) The percentage of cells was attached to each substrate, indicating the ratio of the number of cells remaining on each substrate to the initial number prior to seeding. To quantify the cell number, hNSCs were rinsed twice with PBS after 2 hours of culture and trypsinized; the cell number was manually counted using a hemocytometer. The symbol $*$ indicates a significant difference compared to each $(P<0.005)$. (b) Fluorescence images of hNSCs were adhered to each substrate. After 2 hours of culture, hNSCs were rinsed twice with PBS, and the nuclei were stained with DAPI to visualize the cells on each substrate. The scale bar indicates 10 micrometers.

TABLE 1: RMS roughness values of pNE-modified surfaces determined by AFM analysis.

\begin{tabular}{lcccc}
\hline & PTFE $(\mathrm{nm})$ & PDMS $(\mathrm{nm})$ & Polystyrene $(\mathrm{nm})$ & Glass $(\mathrm{nm})$ \\
\hline Unmodified & $1.239 \pm 0.021$ & $0.091 \pm 0.006$ & $0.138 \pm 0.011$ & $0.257 \pm 0.012$ \\
pNE & $3.391 \pm 0.007^{*}$ & $0.687 \pm 0.032^{*}$ & $0.709 \pm 0.054^{*}$ & $1.119 \pm 0.038^{*}$ \\
\hline
\end{tabular}

The symbol $*$ indicates significant differences compared to each unmodified substrate $(P<0.001)$. 

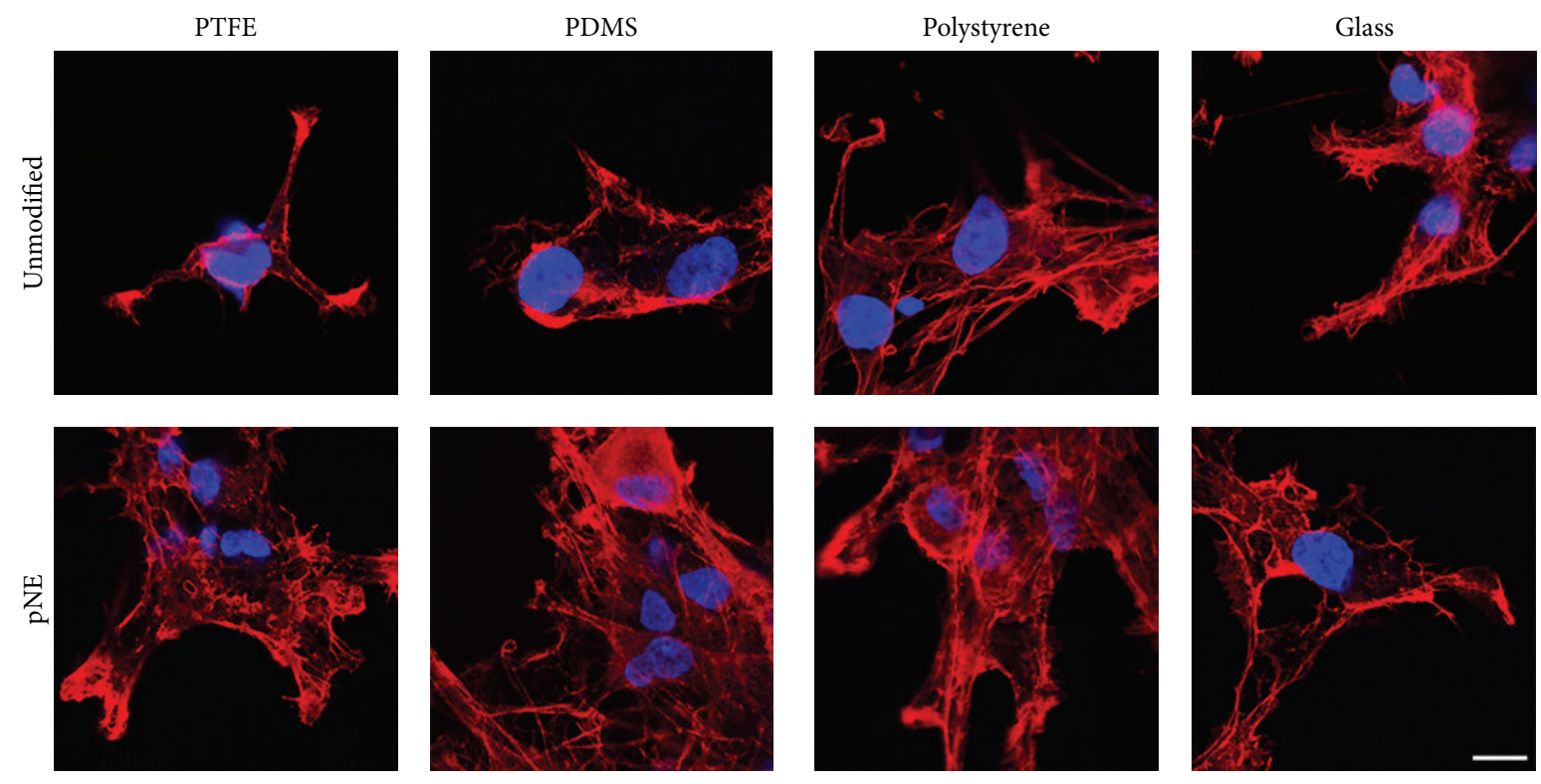

(a)

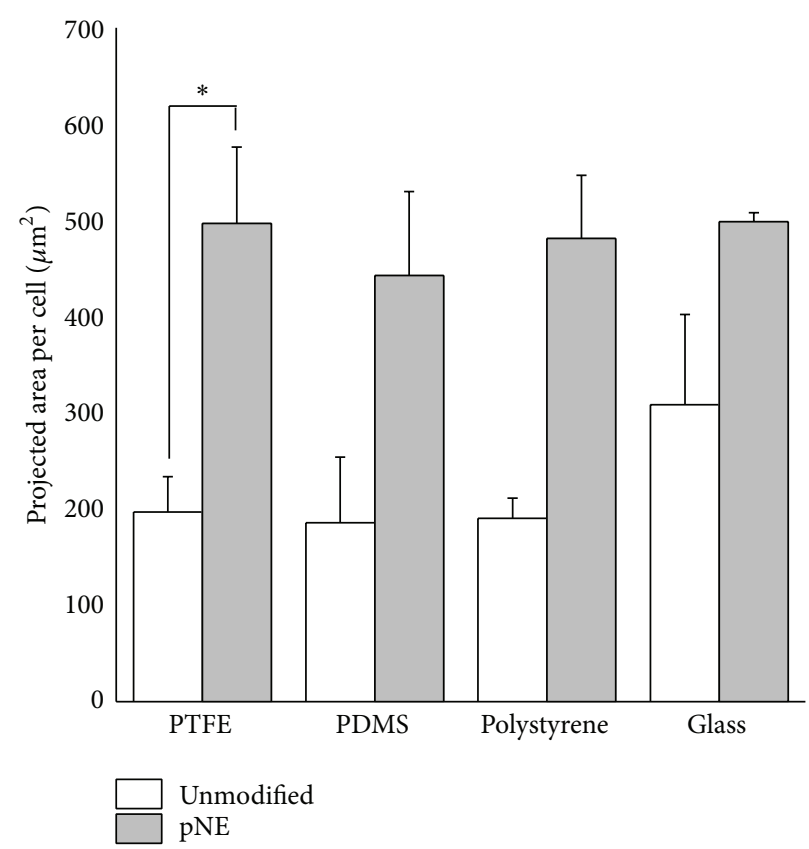

(b)

FIGURE 5: The projected area of hNSCs grown on each substrate. (a) Fluorescence images of actin filaments were stained with rhodaminelabeled phalloidin after 48 hours of culture. The nuclei of hNSCs were stained with DAPI. The scale bar indicates $10 \mu \mathrm{m}$. (b) The total projected areas of the cells were attached to each substrate. Five random images showing actin filaments were acquired, and the total projected areas were quantified using Image J software. The total projected areas were subsequently normalized to the number of cells observed in each image. The symbol ${ }^{*}$ indicates a significant difference compared to the unmodified substrates $(P<0.005)$.

Subsequently, the metabolic activity of hNSCs adhered to each pNE-coated substrate was analyzed to test the potential of $\mathrm{pNE}$ to be employed as a biointerface. Interestingly, the unmodified substrates resulted in no significant reduction in cellular viability compared to that of TCP. Additionally, cellular viability on the pNE-coated substrates at 2,4 , and 6 days of culture was comparable to that of cells grown under normal culture conditions (i.e., cultured on TCP plates) (Figure 3), suggesting the feasibility of the use of pNE interfaces as a cell-culture substrate.

Importantly, the pNE interface on each substrate resulted in the facilitation of cell adhesion (Figure 4). To evaluate the capability of the pNE-coated surfaces to promote cellular attachment, the cells seeded on each substrate were rinsed 

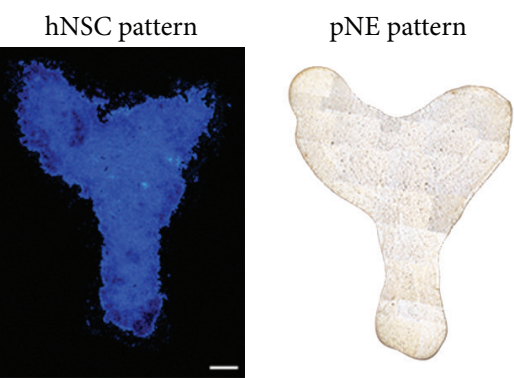

(a)

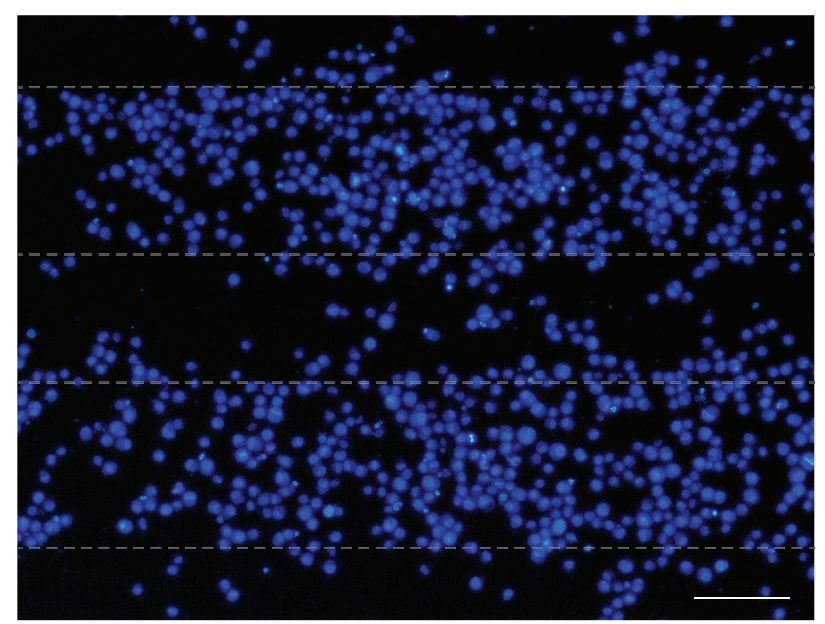

(b)

FIGURE 6: Cellular patterns created using pNE interfaces. (a) Macroscale cellular patterns were created using a micropipette. The "Y" shape of the pNE interfaces was generated with a pipette, and hNSCs were seeded onto the entire TCP substrate. After 2 hours of culture, the loose cells were removed by rinsing with PBS, and the adherent cells were stained with Hoechst dye and imaged using fluorescence microscopy. To visualize the entire cellular pattern, individual images were collected and combined to obtain the entire image. The scale bar indicates $1 \mathrm{~mm}$. (b) Microscale cellular patterns were created with a PDMS stamp. The PDMS stamp was immersed in pNE solution and placed on a TCP plate. The substrate was rinsed with PBS; the cells were seeded onto the entire substrate, rinsed with PBS after 2 hours of culture, and imaged. The nuclei were stained using Hoechst dye. The scale bar indicates 100 micrometers.

twice with PBS after only 2 hours of culture. To measure the enhancement of cellular attachment precisely, the cells remaining on each substrate were trypsinized again and manually counted using a hemocytometer (Figure 4(a)), and the nuclei of the cells remaining on each substrate after rinsing with PBS were visualized (Figure 4(b)). The surface modification with pNE resulted in at least a fourfold enhancement in cellular adherence $(20-25 \%)$ relative to the unmodified naïve material (less than 5\%) $(P<0.005)$, even after the short exposure of the cells to the sticky surface. The ultrasmooth surface caused by the pNE coating might distribute sticky moieties homogeneously throughout the entire surface, thereby potentially causing further intimate interactions with a variety of receptors or proteins residing on the cellular membrane for robust cellular adhesion.

Although the pNE coating substantially increased the surface wettability, the pNE interfaces exhibited enhanced adherent properties. The preferred surface wettability for effective cellular attachment has been reported to be static contact angles ranging from $40^{\circ}$ to $70^{\circ}[26]$. Also, the reasons explaining the enhanced hNSC adhesion are that the pNEcoated surface is abundant in amine groups, which was demonstrated to facilitate stem cell adhesion, for example, polyD-lysine. Another mechanism might be covalent bond formation between cell-surface proteins and pNE. The redox properties of catechol groups in pNE allow protein/peptide/oligonucleotide covalent conjugations [22, 27, 28].

The enhanced interactions of the cellular membrane with the pNE-modified surfaces led to increases in the projected area of adherent cells (Figure 5). The actin filaments were stained with rhodamine-labeled phalloidin (Figure 5(a)), and the total spreading areas of the cells were subsequently normalized to the total cell number (Figure 5(b)). Regardless of the substrate type, the hNSCs adhering to the pNE interfaces exhibited an approximately 1.5- to 6-fold increase in spreading areas per cell compared to the unmodified substrates (Figure 5(b)). Interestingly, marked increases in the projected area per cell adhering to the pNE-coated substrate were observed for the hydrophobic surfaces (i.e., PTFE and PDMS) compared to the unmodified surfaces. The well-stretched actin filaments of cells adhering to pNE interfaces may result from increased surface hydrophilicity [29]. Catecholamine compounds can interact with a variety of proteins or moieties to induce cellular attachment and can subsequently protect cell-surface proteins from the denaturation that can occur upon contact with substrates [29]. The improved stability of the proteins or ligands that have a key role in surface interactions might promote cellular adhesion onto pNE-coated surfaces. Additionally, previous studies have demonstrated that the enhancement of cellular adhesion can alter cytoskeletal development, thereby resulting in increased cell spreading $[29,30]$. This evidence further supports the notion that modulating the cellular microenvironment by manipulating the surface properties of substrates can be critical for elucidating a variety of cellular events, including adhesion, patterning, and proliferation.

The adhesive property of the pNE interfaces enabled the creation of sticky surfaces onto which cells could adhere in a well-defined pattern (Figure 6). Simply drawing the pNE interface with a micropipette followed by cells incubation for 2 hours generated well-defined cellular patterns corresponding to the preformed pNE patterns on a macroscale (Figure 6(a)). The resolution of these cellular patterns was improved using PDMS stamps with straight-line ridges. As shown in Figure 6(b), well-patterned edges between the 
pNE interfaces and nonmodified surfaces were observed, and hNSCs specifically adhered to the pNE patterns. The capability of the pNE interfaces to induce spatial cellular patterns demonstrates their potential as a powerful substrate that can be utilized in tissue engineering applications that require directional cellular growth, such as spinal cord regeneration.

\section{Conclusions}

A simple and facile interface modification strategy to enhance hNSC adhesion and its viability was examined in this study. The surface-modification method is inspired by the neurotransmitter NE, which is oxidatively polymerized in an alkaline solution to generate pNE. pNE becomes an adherent nanolayer during polymerization, resulting in the functionalization of various material surfaces. The pNE interfaces exhibited superior performances in promoting the cellular adhesion of hNSCs, which are recognized as relatively nonadherent primary cells on typical tissue culture plates. The adhered hNSCs exhibited good viability and spreading on these surfaces. Importantly, the adhesive properties of the pNE interfaces led to well-defined patterns of cellular attachment, thereby demonstrating the ability of the cells to organize into micropatterns. Evaluation of the pNE interfaces as a powerful platform to modulate stem cell fates (e.g., proliferation or differentiation) is highly required to be further studied. All of these data indicate that this pNE coating can be a powerful tool to broaden the range of material choices for the ex vivo expansion of hNSCs, an important goal for cell therapy.

\section{Conflict of Interests}

The authors declare that there is no conflict of interests regarding the publication of this paper.

\section{Acknowledgments}

This work was supported by the National Research Foundation of Korea (NRF) Grant through the Active Polymer Center for Pattern Integration (no. 2007-0056091, Jae-Hyung Jang), Basic Science Research Program (2012R1A1A1003397, Jae-Hyung Jang), and Biomedical Technology Development Program (2012-0006085, Haeshin Lee) funded by the Ministry of Science, ICT \& Future Planning (MSIP).

\section{References}

[1] M. D. Mager, V. Lapointe, and M. M. Stevens, "Exploring and exploiting chemistry at the cell surface," Nature Chemistry, vol. 3, no. 8, pp. 582-589, 2011.

[2] A. E. Nel, L. Mädler, D. Velegol et al., "Understanding biophysicochemical interactions at the nano-bio interface," Nature Materials, vol. 8, no. 7, pp. 543-557, 2009.

[3] M. M. Stevens and J. H. George, "Exploring and engineering the cell surface interface," Science, vol. 310, no. 5751, pp. 1135-1138, 2005.
[4] J. Y. Lim and H. J. Donahue, "Cell sensing and response to micro- and nanostructured surfaces produced by chemical and topographic patterning," Tissue Engineering, vol. 13, no. 8, pp. 1879-1891, 2007.

[5] U. Hersel, C. Dahmen, and H. Kessler, "RGD modified polymers: biomaterials for stimulated cell adhesion and beyond," Biomaterials, vol. 24, no. 24, pp. 4385-4415, 2003.

[6] L. Kam, W. Shain, J. N. Turner, and R. Bizios, "Selective adhesion of astrocytes to surfaces modified with immobilized peptides," Biomaterials, vol. 23, no. 2, pp. 511-515, 2002.

[7] Z. Ma, C. Gao, Y. Gong, and J. Shen, "Cartilage tissue engineering PLLA scaffold with surface immobilized collagen and basic fibroblast growth factor," Biomaterials, vol. 26, no. 11, pp. 12531259, 2005.

[8] L. Richert, F. Boulmedais, P. Lavalle et al., "Improvement of stability and cell adhesion properties of polyelectrolyte multilayer films by chemical cross-linking," Biomacromolecules, vol. 5, no. 2, pp. 284-294, 2004.

[9] H. Lee, S. M. Dellatore, W. M. Miller, and P. B. Messersmith, "Mussel-inspired surface chemistry for multifunctional coatings," Science, vol. 318, no. 5849, pp. 426-430, 2007.

[10] K. Y. Suh, J. Seong, A. Khademhosseini, P. E. Laibinis, and R. Langer, "A simple soft lithographic route to fabrication of poly(ethylene glycol) microstructures for protein and cell patterning," Biomaterials, vol. 25, no. 3, pp. 557-563, 2004.

[11] S. M. Kang, I. You, W. K. Cho et al., "One-step modification of superhydrophobic surfaces by a mussel-inspired polymer coating," Angewandte Chemie-International Edition, vol. 49, no. 49, pp. 9401-9404, 2010.

[12] E. Kim, I. T. Song, S. Lee, J. S. Kim, H. Lee, and J. H. Jang, "Drawing sticky adeno-associated viruses on surfaces for spatially patterned gene expression," Angewandte ChemieInternational Edition, vol. 51, no. 23, pp. 5598-5601, 2012.

[13] M. Yamato, C. Konno, M. Utsumi, A. Kikuchi, and T. Okano, "Thermally responsive polymer-grafted surfaces facilitate patterned cell seeding and co-culture," Biomaterials, vol. 23, no. 2, pp. 561-567, 2002.

[14] S. Wang, H. Wang, J. Jiao et al., "Three-dimensional nanostructured substrates toward efficient capture of circulating tumor cells," Angewandte Chemie-International Edition, vol. 48, no. 47, pp. 8970-8973, 2009.

[15] L. Heng, R. Hu, S. Chen, M. Li, L. Jiang, and B. Z. Tang, "Ordered honeycomb structural interfaces for anticancer cells growth," Langmuir, vol. 29, no. 48, pp. 14947-14953, 2013.

[16] X. C. Liu, C. Li, H. Liu et al., "Bio-inspired soft polystyrene nanotube substrate for rapid and highly efficient breast cancercell capture," NPG Asia Materials, vol. 5, p. e63, 2013.

[17] S. H. Ku, J. S. Lee, and C. B. Park, "Spatial control of cell adhesion and patterning through mussel-inspired surface modification by polydopamine," Langmuir, vol. 26, no. 19, pp. 15104-15108, 2010.

[18] H. Lee, J. Rho, and P. B. Messersmith, "Facile conjugation of biomolecu les onto surfaces via mussel adhesive protein inspired coatings," Advanced Materials, vol. 21, no. 4, pp. 431434, 2009.

[19] S. M. Kang, N. S. Hwang, J. Yeom et al., "One-step multipurpose surface functionalization by adhesive catecholamine," Advanced Functional Materials, vol. 22, no. 14, pp. 2949-2955, 2012.

[20] J. H. Waite, "Surface chemistry: mussel power," Nature Materials, vol. 7, no. 1, pp. 8-9, 2008. 
[21] C. R. Nuttelman, D. J. Mortisen, S. M. Henry, and K. S. Anseth, "Attachment of fibronectin to poly(vinyl alcohol) hydrogels promotes NIH3T3 cell adhesion, proliferation, and migration," Journal of Biomedical Materials Research, vol. 57, no. 2, pp. 217223, 2001.

[22] M. K. Sung, J. Rho, I. S. Choi, P. B. Messersmith, and H. Lee, "Norepinephrine: material-independent, multifunctional surface modification reagent," Journal of the American Chemical Society, vol. 131, no. 37, pp. 13224-13225, 2009.

[23] S. M. Kang and H. Lee, "Surface Modification of Highly Ordered Pyrolytic Graphite (HOPG) by a mussel-inspired Poly(norepinephrine) coating: characterizations and cell adhesion test," Bulletin of the Korean Chemical Society, vol. 34, no. 3, pp. 960-962, 2013.

[24] S. Hong, J. Kim, Y. S. Na et al., "Poly(norepinephrine): ultrasmooth material-independent surface chemistry and nanodepot for nitric oxide," Angewandte Chemie-International Edition, vol. 52, no. 35, pp. 9187-9191, 2013.

[25] H.-T. Kim, I.-S. Kim, I.-S. Lee, J.-P. Lee, E. Y. Snyder, and K. I. Park, "Human neurospheres derived from the fetal central nervous system are regionally and temporally specified but are not committed," Experimental Neurology, vol. 199, no. 1, pp. 222235, 2006.

[26] Y. Arima and H. Iwata, "Effect of wettability and surface functional groups on protein adsorption and cell adhesion using well-defined mixed self-assembled monolayers," Biomaterials, vol. 28, no. 20, pp. 3074-3082, 2007.

[27] K. Yang, J. S. Lee, J. Kim et al., "Polydopamine-mediated surface modification of scaffold materials for human neural stem cell engineering," Biomaterials, vol. 33, no. 29, pp. 6952-6964, 2012.

[28] H. O. Ham, Z. Liu, K. H. A. Lau, H. Lee, and P. B. Messersmith, "Facile DNA immobilization on surfaces through a catecholamine polymer," Angewandte Chemie-International Edition, vol. 123, no. 3, pp. 758-762, 2011.

[29] S. H. Ku, J. Ryu, S. K. Hong, H. Lee, and C. B. Park, "General functionalization route for cell adhesion on non-wetting surfaces," Biomaterials, vol. 31, no. 9, pp. 2535-2541, 2010.

[30] K. Anselme, "Osteoblast adhesion on biomaterials," Biomaterials, vol. 21, no. 7, pp. 667-681, 2000. 

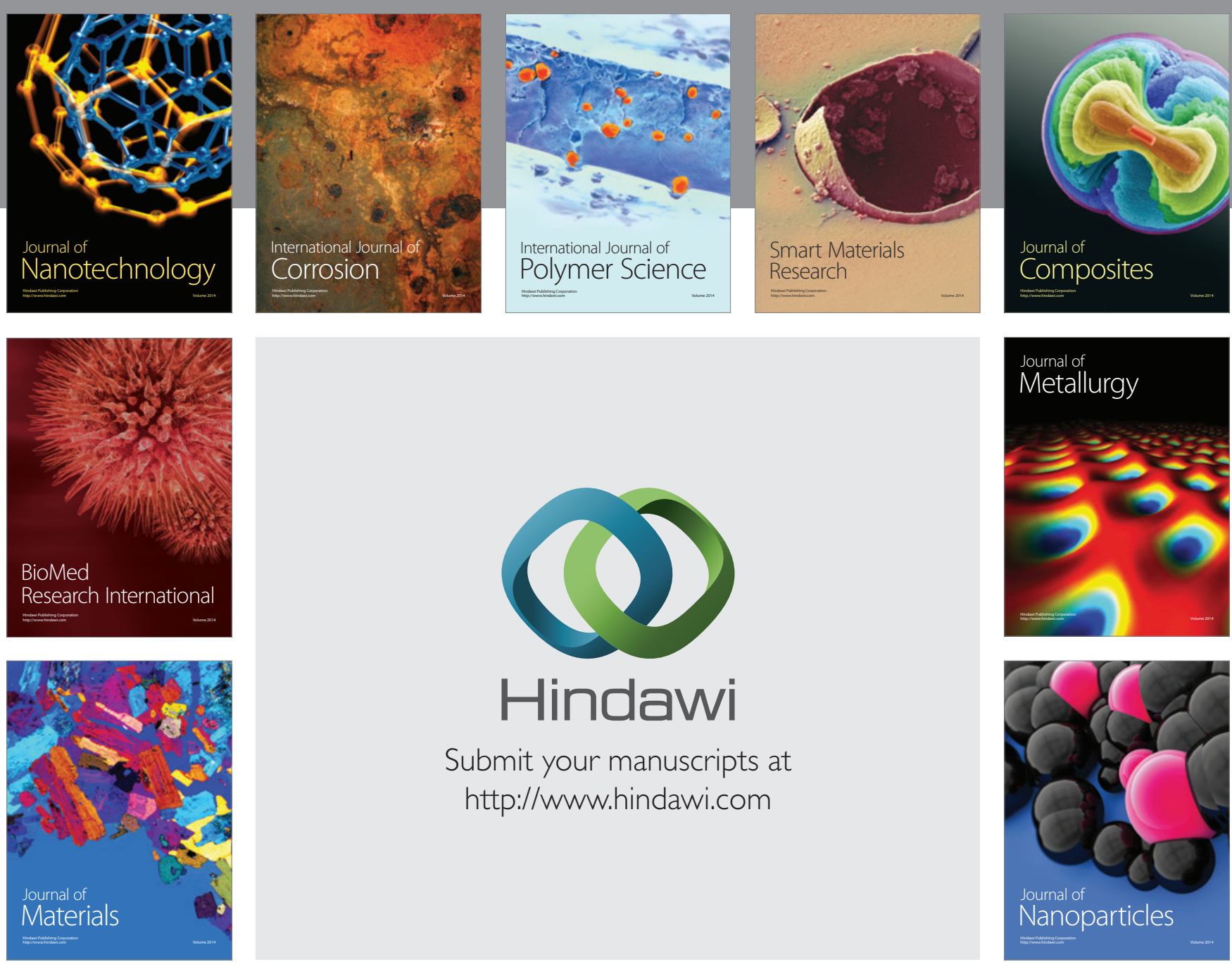

Submit your manuscripts at http://www.hindawi.com
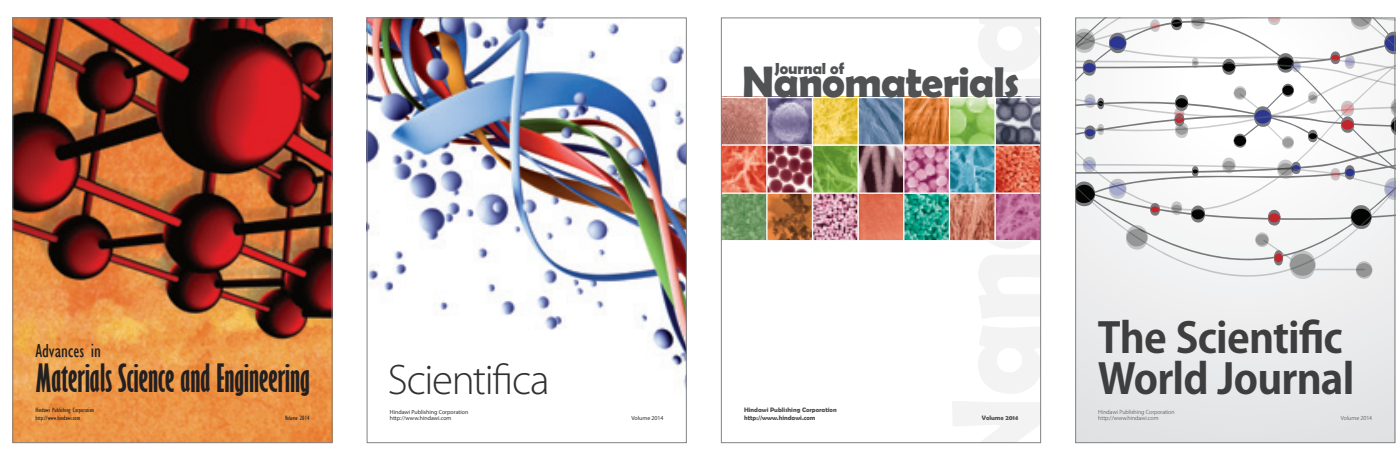

\section{The Scientific World Journal}
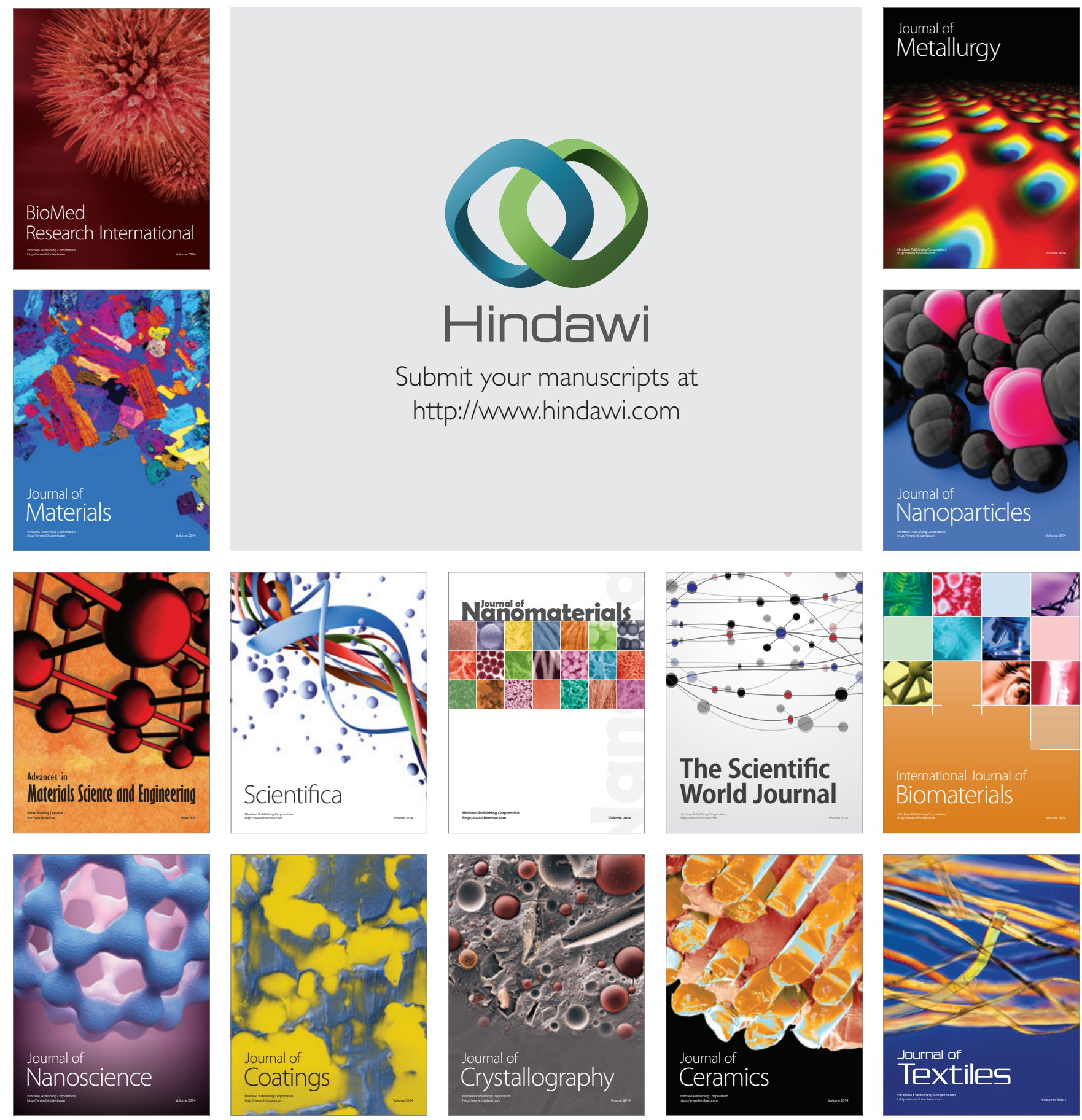